(non-bypass (LVO)). Population-weighted median and interquartile range were calculated for each at the national level. Results 72,539 census tracts, 2,388 certified stroke hospitals, and 371 certified EVT-capable centers were included. The population-weighted median distance for non-bypass (nonLVO), bypass, and non-bypass (LVO) routing was $8.5 \mathrm{~km}$ (IQR: 4.6, 18.2), $24.8 \mathrm{~km}$ (IQR: 10.4, 69.3), and $31.1 \mathrm{~km}$ (IQR: 13.4, 83.3), respectively. The population-weighted median difference in between bypass and non-bypass routing was $11.8 \mathrm{~km}(1.8,42.8)$ for non-LVO patients and $3.2 \mathrm{~km}$ $(0.4,9.9)$ for LVO patients.

Conclusions Bypass incurs a modest distance penalty for nonLVO patients and offers a modest distance benefit for LVO patients. These differences in distance are likely insignificant to intra-hospital delays that contribute greatly to delays in EVT treatment in non-bypass transport. Based on current guidelines, bypass of the nearest stroke center for an EVTcapable center may be warranted for a majority of Americans. Disclosures C. Yu: None. P. Panagos: None. A. Kansagra: None.

\section{E-103 PEDIATRIC HOSPITAL PROXIMITY TO ENDOVASCULAR THROMBECTOMY CENTERS IN THE UNITED STATES}

${ }^{1} \mathrm{C} \mathrm{Yu}{ }^{*},{ }^{1} \mathrm{~K}$ Guilliams, ${ }^{1} \mathrm{P}$ Panagos, ${ }^{1,2} \mathrm{~A}$ Kansagra. ${ }^{1}$ Washington University School of Medicine, St. Louis, MO; ${ }^{2}$ Mallinckrodt Institute of Radiology, St. Louis, MO

\subsection{6/neurintsurg-2021-SNIS.198}

Introduction Endovascular thrombectomy is an emerging, timesensitive intervention for children with acute ischemic stroke, but this treatment is not commonly available within pediatric hospitals. Access to this therapy often requires inter-hospital transfer to an adult thrombectomy center. Although many pediatric hospitals employ this model of care, the proximity of pediatric hospitals to thrombectomy centers has not previously been characterized. This cross-sectional study quantifies the distance and travel time between pediatric hospitals and their nearest thrombectomy centers by road and aerial routes throughout the contiguous United States to determine the feasibility of transferring pediatric patients for thrombectomy.

Materials and Methods We included pediatric acute care hospitals from the Children's Hospital Association database and thrombectomy centers certified by a national or state organization in the contiguous United States. Distances from each pediatric hospital to its nearest thrombectomy center were calculated using road networks from a public mapping service for road routes and spherical geodesic distance for aerial routes. Road travel times were estimated by dividing road distance by two different average speeds to reflect travel on both smaller roads and highways. Air travel times were estimated by dividing aerial distance by the average speed of the most commonly used medical helicopters. The main outcome is travel time from pediatric hospitals to their nearest thrombectomy centers.

Results 202 pediatric acute care hospitals and 371 thrombectomy centers were included. Median road and aerial distance from a pediatric hospital to the nearest thrombectomy center was $0.80 \mathrm{~km}$ and $0.35 \mathrm{~km}$, respectively. At a road speed of $65 \mathrm{~km} / \mathrm{hr}$ (about $40 \mathrm{mi} / \mathrm{hr}$ ), 182 (90\%) and 183 (91\%) pediatric hospitals were within 30 minutes and 60 minutes, respectively, of a thrombectomy center. By air, 187 (93\%) and 199
(99\%) pediatric hospitals were within 30 minutes and 60 minutes of a thrombectomy hospital.

Conclusions The vast majority of pediatric hospitals are located near thrombectomy care. These results suggest that partnerships between pediatric hospitals and thrombectomy centers can assure pediatric access to thrombectomy without unreasonably lengthy transfers.

Disclosures C. Yu: None. K. Guilliams: None. P. Panagos: None. A. Kansagra: None.

\section{E-104 YOUNG MAN WITH OSTEOGENESIS IMPERFECTA PRESENTING WITH ACUTE STROKE FROM CAROTID DISSECTION AND SUBSEQUENT CONTRALATERAL PSEUDOANEURYSM}

A Mbaluka, R Sanchez-Mejia, R Ryan*. Neurosurgery, Virginia Mason Medical Center, Seattle, WA

\subsection{6/neurintsurg-2021-SNIS. 199}

Background Osteogenesis Imperfecta (OI) is a hereditary disease that affects connective tissues causing symptoms such as bone fragility, among other connective tissue ailments. OI has four primary classifications: dominant inherited with blue sclerae, lethal prenatal, progressively deforming and dominantly inherited with normal sclerae. The connective tissue disease has been primarily associated with dominant autosomal mutations in collagen coding genes COL1A1 and COL1A2. Likely related to the collagen defects, these patients are at risk for vessel dissection with sequalae related to the affected vessel and degree of injury.

Methods We describe the case of a 33 year-old man with autosomal dominant hereditary OI that presented with acute stroke from left cervical carotid artery dissection causing occlusion. He had significant right sided deficits that persisted despite administration of TPA and he was taken for emergent endovascular intervention.

Results Cerebral angiography showed poor collateral circulation to the left hemisphere, and complete occlusion of the cervical left internal carotid artery from dissection, with the distal vessel supplied by small external carotid collaterals

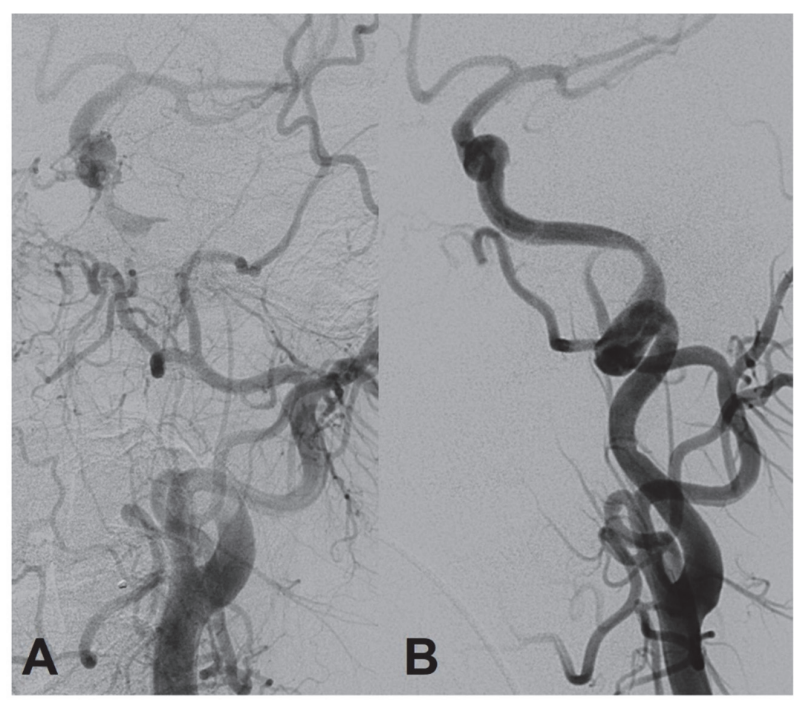

Abstract E-104 Figure 1 
(figure 1a). He was treated with emergency stenting using multiple overlapping devices to reconstruct the vessel lumen and restore anterograde flow (figure $1 b$ ). He made full neurologic recovery and was maintained on dual antiplatelet therapy. Despite maintenance therapy, follow up imaging 3 months later demonstrated development of a new high-grade dissecting pseudoaneurysm of the contralateral right cervical carotid artery that was subsequently treated with stent reconstruction on elective basis, with satisfactory result. Further follow up imaging demonstrated maintained patency of the bilateral stent constructs and he remained symptom free.

Discussion/Conclusion Osteogenesis imperfecta (OI) has been associated with numerous cerebrovascular conditions including carotid cavernous fistulas, cerebral aneurysms, and carotid dissections with attendant risk of stroke. While our patient was able to be successfully treated for his initial carotid dissection in the acute phase, he was at higher risk for stroke, whereas his subsequent carotid dissection was identified pre-symptomatically and treated electively. Judicious evaluation of patients with OI for cervical carotid dissections is warranted for early identification and treatment.

Disclosures A. Mbaluka: None. R. Sanchez-Mejia: None. R. Ryan: None.

\section{E-105 ANALYSIS OF CLINICAL ASSOCIATIONS OF SERUM SCFAS TAKEN AT THE TIME OF THROMBECTOMY IN AIS PATIENTS}

${ }^{1} \mathrm{~N}$ Henry* ${ }^{2}{ }^{2} \mathrm{~J}$ Frank, ${ }^{3} \mathrm{~J}$ Fraser, ${ }^{2} \mathrm{~A}$ Trout, ${ }^{4} \mathrm{~A}$ Morris, ${ }^{5} \mathrm{~J}$ Chen, ${ }^{6} \mathrm{~A}$ Stowe, ${ }^{7} \mathrm{C}$ McLouth, ${ }^{6} \mathrm{~K}$ Pennypacker. ${ }^{1}$ College of Medicine, University of Kentucky, Lexington, KY; ${ }^{2}$ Neurology, Center for Advanced Translational Stroke Science, University of Kentucky, Lexington, KY; ${ }^{3}$ Neurology, Neurosurgery, Radiology, Neuroscience, Center for Advanced Translational Stroke Science, University of Kentucky, Lexington, KY; ${ }^{4}$ Pharmacology and Nutritional Sciences, University of Kentucky, Lexington, KY; ${ }^{5}$ Neurology, University of Kentucky, Lexington, $K Y_{;}{ }^{6}$ Neurology, Neuroscience, Center for Advanced Translational Stroke Science,

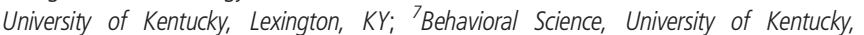
Lexington, $K Y$

\subsection{6/neurintsurg-2021-SNIS.200}

Introduction Gut dysbiosis has become a well-recognized process that occurs following stroke, which may provide clinically relevant implications in stroke severity and patient outcome. Short chain fatty acids (SCFAs) are gut microbiota-derived metabolites that contribute to the gut-brain axis and have shown growing importance in stroke recovery and patient functional outcomes. However, the systemic role of these molecules at the time of thrombectomy immediately following stroke and their risk factors have not been well explored.

Methods Blood and Clot Thrombectomy Registry and Collaboration (BACTRAC), a continuously enrolling tissue bank, was used to obtain stroke samples. Arterial blood distal and proximal to the thrombus was obtained from Acute Ischemic Stroke (AIS) Patients $(n=81)$, and plasma was immediately isolated and frozen. Patient demographics, stroke severity, and functional outcomes were also recorded. The concentrations of the SCFAs: Acetate, Propionate, Isobutyrate, Butyrate and 2-Methylbutyrate were quantified from the proximal plasma samples via chemical derivatization Ultra-High Performance Liquid Chromatography (UHPLC) coupled tandem mass spectrometry using electrospray ionization and multiple reaction monitoring. Bivariate analysis and two-tailed t-testing of the patient parameters with the SCFA concentrations were computed to identify results. Significance was set at $\mathrm{P}<=0.05$.
Results Our analysis found serum concentrations of the SCFAs, with the exception of Acetate, were all positively correlated with patient age. Propionate, Isobutyrate, and 2-Methylbutyrate were only positively correlated with age in males but not females. Interestingly, Propionate, Isobutyrate, Butyrate and 2Methylbutyrate were positively correlated with each other, but not with Acetate. Acetate, Isobutyrate, and 2-Methylbutyrate were positively correlated with the time from last known normal to thrombectomy (minutes), suggesting a temporal aspect in the levels of these molecules following stroke. Patients who never smoked were found to have significantly higher concentrations of Isobutyrate $(p=0.009)$, Butyrate $(p=0.023)$, and 2Methylbutyrate $(p=0.045)$ than those who were current smokers. In women only, higher Isobutyrate concentrations were correlated with more severe patient disability at the time of stroke, and with worse functional outcomes at discharge. Beyond this finding, stroke severity (i.e. infarct volume) and functional outcome parameters (i.e. NIHSS score) were not found to be associated with SCFAs in this study.

Conclusions The evaluation of SCFAs in the serum at the time of stroke provides novel insight into the potential role of SCFAs during stroke in human patients. Age remains a significant factor to SCFA concentrations in stroke patients. The length of time following stroke also contributes to the levels of SCFAs. Previous studies have shown that increased levels of SCFAs is associated with decreased severity of stroke, however our data did not detect any such effect. This observation may be due to the acute time of the blood collection following stroke and analyzing serum instead of fecal samples. Furthermore, acquiring information about variations in diet and gut microbiota between patients may help explain inter-individual differences in baseline SCFA levels. The quantification of serum SCFAs prior to thrombectomy warrants additional analysis to determine their early role in the systemic response to stroke.

Disclosures N. Henry: None. J. Frank: None. J. Fraser: None. A. Trout: None. A. Morris: None. J. Chen: None. A. Stowe: 1; C; American Heart Association, NIH Grants. 6; C; Cerelux, LLC. C. McLouth: None. K. Pennypacker: 6; C; Cerelux, LLC.

\section{E-106 TIMELINE OF OCCLUSION FOR INTRACRANIAL ANEURYSMS TREATED WITH PIPELINE EMBOLIZATION DEVICES}

S Cler ${ }^{*}$, D Lauzier, A Kansagra. Washington University School of Medicine, St. Louis, MO

\subsection{6/neurintsurg-2021-SNIS.201}

Introduction/Purpose The Pipeline Embolization Device (PED) is commonly used to treat intracranial aneurysms. The rate of aneurysm occlusion at pre-specified timepoints after PED treatment is known from clinical trials, but the actual time evolution of aneurysm occlusion is not known. In this study, we take advantage of real-world variability in the timing of angiographic follow up to characterize the time evolution of aneurysm occlusion.

Materials and Methods Data from a large, urban, tertiary care center was retrospectively analyzed. Only saccular, unruptured aneurysms treated with PED were included in this study. Aneurysm occlusion over time was characterized with KaplanMeier analysis. Aneurysms were censored after angiographic confirmation of complete aneurysm occlusion. Aneurysms were 